\title{
Level Set Based Surface Capturing in 3D Medical Images
}

\author{
Bin Dong ${ }^{1}$, Aichi Chien ${ }^{2}, \mathrm{Yu} \mathrm{Mao}^{1}$, Jian $_{\mathrm{Ye}^{1}}$, and Stanley Osher ${ }^{1}$ \\ ${ }^{1}$ Department of Mathematics, University of California, Los Angeles, CA \\ ${ }^{2}$ Division of Interventional Neuroradiology, David Geffen School of Medicine at \\ UCLA, 10833 LeConte Ave, Los Angeles, CA*
}

\begin{abstract}
Brain aneurysm rupture has been reported to be directly related to the size of aneurysms. The current method used to determine aneurysm size is to manually measure the width of the neck and height of the dome on a computer screen. Because aneurysms usually have complicated shapes, using the size of the aneurysm neck and dome may not be accurate and may overlook important geometrical information. In this paper we present a level set based illusory surface algorithm to capture the aneurysms from the vascular tree. Since the aneurysms are described by level set functions, not only the volume but also the curvature of aneurysms can be computed for medical studies. Experiments and comparisons with models used for capturing illusory contours in 2D images are performed. This includes applications to clinical image data demonstrating the procedure of accurately capturing a middle cerebral artery aneurysm.
\end{abstract}

\section{Introduction}

Subarachnoid hemorrhage, primarily from brain aneurysm rupture, accounts for 5 to $10 \%$ of all stroke cases with a high fatality rate [1. Advancements in neuroimaging technology have helped these aneurysms to be more frequently found prior to rupture. A method to determine if aneurysms are at higher risk of rupturing would be extremely valuable. Brain aneurysm rupture has been reported to be related to the size of aneurysms. It is known that the risk of rupture greatly increases as the aneurysm becomes larger [2. Currently, methods to determine the aneurysmal size are to manually measure the size of the neck and the dome of aneurysms. However, these method may overlook important geometric information [3].

Our goal in this paper is to segment the aneurysm from the entire blood vessel with minimal human interaction. We observe from Fig. 11(d) and 1)(e) that the problem can be realized as an illusory surface capturing problem, as an extension from illusory contours in 2D. Illusory contours have been intensively studied in cognitive neuroscience noting that human vision system is capable of combining nonexistent edges and making meaningful visual organization of both

\footnotetext{
* The research is supported by NIH GRANT, P20 MH65166; NSF GRANT, DMS0714807.
}

D. Metaxas et al. (Eds.): MICCAI 2008, Part I, LNCS 5241, pp. 162 169, 2008.

(C) Springer-Verlag Berlin Heidelberg 2008 
the real and imaginary contour segments (e.g. the Kanizsa square in Fig. 1(e)). Various researchers have introduced mathematical models and techniques to mimic the human vision system in detecting and capturing perceptional contours in images 456]. These mathematical models can be used to describe the process in clinical evaluation when the location of an aneurysm needs to be identified (e.g. Fig. 1(b)). Given that our problem is to first capture and then calculate the volume and geometry of the aneurysm, representing the surface using a level set function and designing a proper surface evolution PDE is essential. In this paper, we introduce a level set and PDE based illusory surface model, inspired by the illusory contour model [6], to calculate the volume and geometry of aneurysms.

\section{Method}

The focus of this paper is to introduce a novel method to capture a specific part of a given surface obtained from 3D images. Therefore, we will not place emphasis on the techniques of $3 \mathrm{D}$ reconstruction. Interested readers can consult 78/9 for details on surface reconstruction. As as an example, we applied a simple thresholding method followed by fast sweeping method [10] and nonlocal means surface smoothing 9] to reconstruct the surface represented by a level set function [11, which takes positive values inside the vessel region and negative values outside (Fig. 11(a)).

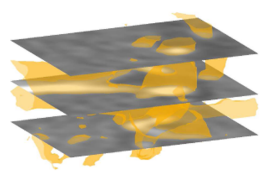

(a)

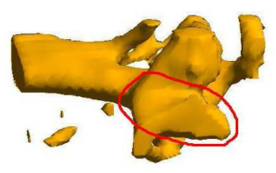

(b)

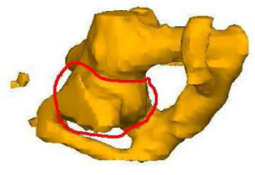

(c)

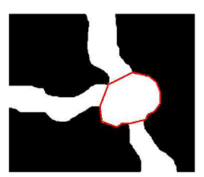

(d)

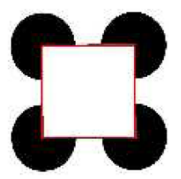

(e)

Fig. 1. (a) is an illustration of surface reconstruction using CT images. (b) and (c) are illustrations to show the location of the aneurysm (red curves) from different viewpoints. (d) is the phantom vessel image where the red curve is the illusory contour. (e) is the Kanizsa square where the red curve is the illusory contour.

\subsection{Level Set Based Illusory Surface Capturing}

Similar to the model introduced by Zhu et al 6] which captures illusory contours in $2 \mathrm{D}$, we consider the following energy function in $3 \mathrm{D}$

$$
E(\phi)=\int_{\Omega}(d \delta(\phi)|\nabla \phi|+\alpha H(\psi) H(\phi)+\beta \delta(\phi)|\nabla \phi|) d x
$$

where $\psi$ is the signed distance function obtained from the previous section and $d=|\psi|$ is the corresponding unsigned distance function. The symbol $\nabla$ is the gradient operator, $\delta(\phi)$ is the Dirac delta functional, and $H(\phi)$ is the Heaviside function. The energy term $\alpha H(\psi)$ acts as a barricade for $\phi$. It forces the evolving 
zero level set of $\phi$ to stay inside of that of $\psi$, and it is inactive when the zero level set of $\phi$ is already inside $\psi$. As a result, the parameter $\alpha$ is less important than $\beta$.

From equation (2.1), the corresponding gradient flow can be written as

$$
\frac{\partial \phi}{\partial t}=\delta(\phi) \nabla d \cdot \frac{\nabla \phi}{|\nabla \phi|}+\delta(\phi) d \nabla \cdot \frac{\nabla \phi}{|\nabla \phi|}-\alpha \delta(\phi) H(\psi)+\beta \delta(\phi) \nabla \cdot \frac{\nabla \phi}{|\nabla \phi|} .
$$

Since the function $\delta(\phi)$ is concentrated only on the zero level set of $\phi$, the PDE (2.2) only describes a motion for the zero level set of $\phi$. Similar to [12, to ensure all level sets of $\phi$ have similar motions and to be able to solve the PDE on the entire 3D rectangular domain, we replace $\delta(\phi)$ in (2.2) by $|\nabla \phi|$ and obtain the following PDE

$$
\frac{\partial \phi}{\partial t}=|\nabla \phi|\left(\nabla d \cdot \frac{\nabla \phi}{|\nabla \phi|}+d \nabla \cdot \frac{\nabla \phi}{|\nabla \phi|}-\alpha H(\psi)+\beta \nabla \cdot \frac{\nabla \phi}{|\nabla \phi|}\right) .
$$

Equation (2.3) is the illusory contour model proposed in [6] with a direct application in 3D. In that paper, the authors also considered the following improved model which enables the final curves to stick to sharp corners more closely

$$
E(\phi)=\int_{\Omega}\left(\left(1+\nu c_{a, b} \kappa^{+}(\psi)\right) d \delta(\phi)|\nabla \phi|+\alpha H(\psi) H(\phi)+\beta \delta(\phi)|\nabla \phi|\right) d x,
$$

where $\nu$ is some constant, $c_{a, b}$ is some restriction function defined in (2.7) and $\kappa^{+}(\psi)$ is the positive part of curvature. However, a direct extension of (2.4) to 3D (using Gaussian curvature for $\kappa$ ) does not give satisfactory results. A detailed discussion and comparisons will be given in Section 3.1 .

\subsection{Modified Illusory Surface Model}

Here we introduce our modified illusory surface model based on equation (2.3). We observe that the dominant force $\nabla d$ in (2.3) does not distinguish between the relatively flat regions and the sharp tip on the aneurysm (Fig. 1). Therefore, we introduce an amplification factor in front of $\nabla d \cdot \frac{\nabla \phi}{|\nabla \phi|}$ (shown in (2.5) ) in order to handle problems with complicated geometry, for example the geometry with extruded bleb. It is somehow similar to [6, but instead of putting the factor in the energy as it is in (2.4), we modify (2.3). The amplification factor, denoted as $A(\psi)$, and the modified PDE are given as follows

$$
\begin{gathered}
\frac{\partial \phi}{\partial t}=|\nabla \phi|\left(A(\psi) \nabla d \cdot \frac{\nabla \phi}{|\nabla \phi|}+d \nabla \cdot \frac{\nabla \phi}{|\nabla \phi|}-\alpha H(\psi)+\beta \nabla \cdot \frac{\nabla \phi}{|\nabla \phi|}\right), \\
A(\psi)=1+\mu \kappa^{+}(\psi),
\end{gathered}
$$

where $\mu$ is a constant parameter and $\kappa^{+}(\psi)$ is the positive part of the Gaussian curvature of $\psi$. By using the Gaussian curvature, we can automatically distinguish the target region (aneurysm) from the blood vessels, because both 
aneurysm and vessel regions have comparable values of mean curvatures, while only in the aneurysm region, especially the sharp tip (Fig. 1), is the Gaussian curvature large. Our experiments show that our model (2.5) performs better than (2.3) and (2.4), especially in the region with high Gaussian curvature. Numerical results and a discussion of our model (2.5), and the model (2.3) and (2.4) can be found in Section 3.1.

\subsection{Numerical Procedure and Computations of Geometries}

Since the energy functional (2.1) is not convex and, indeed, it has many local minimizers, in order to obtain a desirable solution for equation (2.3), good choices of $\phi(x, 0)$ are needed [6]. For our model (2.5), a reasonable initialization is also required. However, our model (2.5) is less restrictive on initial guesses than (2.3). This is tested and discussed in detail in the numerical section. To obtain a reasonable initial surface, we need users to select points around the area of interest and initiate the computation. We use the selected points to determine a sphere with level set function $\phi_{\mathrm{S}}$. Then $\phi(x, 0)$ is defined as the intersection of $\phi_{\mathrm{S}}$ with $\psi$, or mathematically $\phi(x, 0)=\min \left\{\phi_{\mathrm{S}}(x), \psi(x)\right\}$. In our proposed method, the selection of the points for the region of interest is the only part needs users interaction. Although automated computation is desirable, determining a pathologic region is a medical diagnosis which needs an expert's supervision. Therefore, it is reasonable to have experts' inputs and use them to initiate the computation. In the result section, we will show a clinical example of allowing the user to select as few as six points to capture the surface of a brain aneurysm.

With the initial condition described above, we employ the local level set method [13] to solve equation (2.3) and (2.5), as well as to minimize (2.4), in order to alleviate the time step restrictions and lower the complexity of our numerical computations. Generally speaking, we solve $\phi_{t}+c_{a, b}(\phi) V_{n}(\phi)|\nabla \phi|=0$ instead of $\phi_{t}+V_{n}(\phi)|\nabla \phi|=0$ with the restriction function $c_{a, b}$ introduced to confine all effective calculations within a narrow band of zero level set of $\phi$. The restriction function $c_{a, b}$ is defined as

$$
c_{a, b}(x)= \begin{cases}1, & |x| \leq a \\ (|x|-b)^{2}(2|x|+b-3 a) /(b-a)^{3} & , a<|x| \leq b \\ 0, & |x|>b\end{cases}
$$

After we obtain the solution $\phi$ which represents the aneurysm, we calculate the volume of it by $V(\phi)=\int H(\phi) d x$, the mean curvature by $\kappa_{m}(\phi)=\nabla \cdot \frac{\nabla \phi}{|\nabla \phi|}$, and the Gaussian curvature [14] by $\kappa_{\mathrm{g}}(\phi)=\frac{\nabla \phi^{T} H(\phi) \nabla \phi}{|\nabla \phi|^{4}}$, where

$$
H(\phi)=\left(\begin{array}{ccc}
\phi_{y y} \phi_{z z}-\phi_{y z} \phi_{z y} & \phi_{y z} \phi_{z x}-\phi_{y x} \phi_{z z} & \phi_{y x} \phi_{z y}-\phi_{y y} \phi_{z x} \\
\phi_{x z} \phi_{z y}-\phi_{x y} \phi_{z z} & \phi_{x x} \phi_{z z}-\phi_{x z} \phi_{z x} & \phi_{x y} \phi_{z x}-\phi_{x x} \phi_{z y} \\
\phi_{x y} \phi_{y z}-\phi_{x z} \phi_{y y} & \phi_{y x} \phi_{x z}-\phi_{x x} \phi_{y z} & \phi_{x x} \phi_{y y}-\phi_{x y} \phi_{y x}
\end{array}\right)
$$

and subscripts denote the partial derivatives in Cartesian coordinates. 


\section{Applications in Medical Images}

The algorithms are applied to brain images acquired by 3D CT angiography. The images have $512 \times 512$ in-plan spatial resolutions with each voxel size approximately $0.125 \mathrm{~mm}^{3}$. We then extract subimages of size $54 \times 37$ for the aneurysm from the entire brain images.

\subsection{Numerical Experiments and Validations}

The numerical experiments were performed using MATLAB. It took approximately 90 seconds to capture the aneurysm surface using a Windows Laptop (Duo processor, 2.0GHz CPU and 2GB RAM). The numerical stopping criteria for the iteration $\phi^{n} \rightarrow \phi^{n+1}$ is based on $\frac{\left\|\phi^{n+1}-\phi^{n}\right\|_{2}}{\left\|\phi^{n}\right\|_{2}}<$ tolerance.

The numerical results of solving (2.3) are shown in Fig. 2, row one. Although this model has been reported with fairly good results for 2D images [6], direct application to capturing $3 \mathrm{D}$ surface is not satisfactory. The reason is that in 6 , successful contour/surface capturing highly depends on the choice of an initial curve/surface which should cover all regions of interests. However, given that our problem is to capture a complicated surface with a few initial points, it is difficult to ensure that the assigned initial surface provides good coverage. Also, a larger initial surface will result in significant increase in the computational time. Therefore, the model (2.3) is not suited for solving our problem.

We also test the model (2.4) which was developed to improve the illusory contours at corners [6]. The results are shown in the second row of Fig. 2, This model provides some improvement at the tip of the aneurysm in comparison with the model (2.3). However, it still can not capture the entire tip which is a very

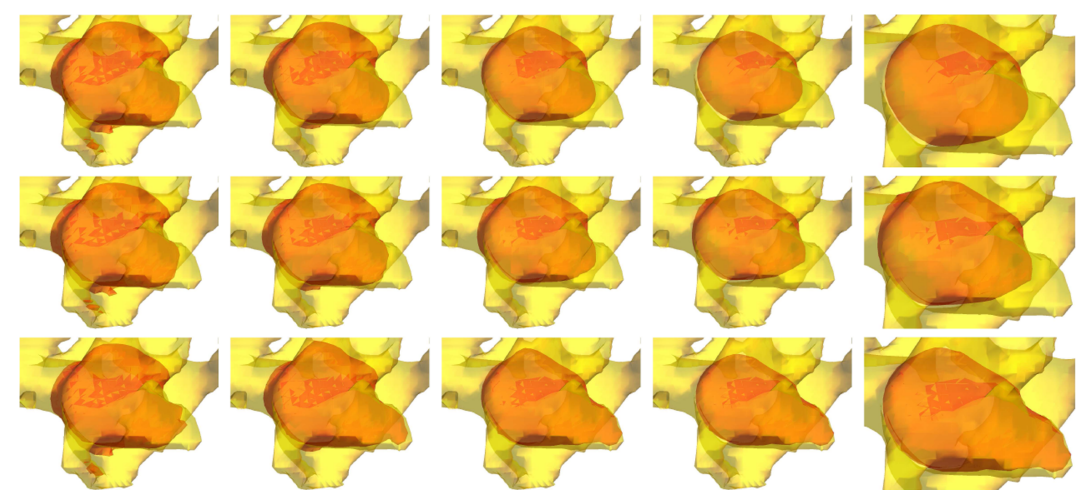

Fig. 2. Row 1-3 shows results of (2.3), (2.4) and (2.5) respectively. For the best results, parameters are $\beta=1$ for (2.3), $(\mu, \beta)=(500,0.05)$ for (2.4) and $(\mu, \beta)=(2000,1)$ for (2.5). In each row, the first four figures are results at iteration=20, 100, 500 and 1000 respectively, and the last figure is the magnified view at iterations $=1000$. All the initial points are given as the same as the first column in Fig. 5. 


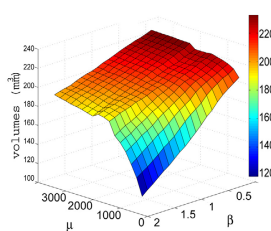

(a)

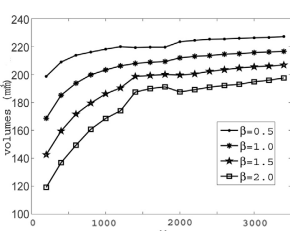

(b)

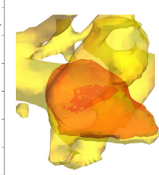

(c)

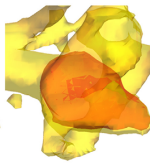

(d)

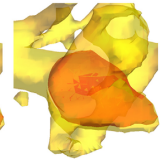

(e)

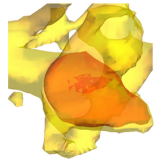

(f)

Fig. 3. (a) and (b) show the influence of $(\mu, \beta)$ to the captured volume. (c)-(f) show the visualization of the final surface capturing with parameters $\mu=2000$, and $\beta=$ $0.5,1,1.5$ and 2 respectively. (d) shows the best surface capturing.

important medical feature. The reason is that fundamentally the illusory surface model (2.4) creates a boundary near the region where the concavity changes. For the aneurysm we have here, the concavity changes near the sharp tip (Fig. (2)). Therefore, 2.4 can not capture the entire aneurysm.

The results of our modified surface capturing model 2.5) are shown in the third row of Fig. 2. Using this method, we are able to capture the entire aneurysm. The reason for this significant improvement is that our modification is made directly on the force field (the factor $A(\psi) \nabla d$ in the first term of (2.5) ), which guides the movement of the zero level set of $\phi$ towards the part of the surface with high Gaussian curvature. However, other terms in the original PDE (2.3) are kept unchanged, especially the second term $d \nabla \cdot \frac{\nabla \phi}{|\nabla \phi|}$, in contrast to the corresponding PDE of (2.4) 6].

\subsection{Parameter Comparison and Geometry Computation}

Since parameters $\beta$ and $\mu$ in (2.5) and (2.6) can both affect the final results, we experiment on how they interact with each other and affect the quality of the captured surface and volume. Fig. 3(a) shows that the choice of $\beta$ significantly changes the captured volume. In addition, the volume increases considerably with $\mu$ until it reaches 2000 (Fig. 3(b)). After that stage the captured volume does not change too much (approximately $2 \mathrm{~mm}^{3}$ ). In general, the larger $\beta$ is, the smoother the final surface will be; however, we tend to lose the tip when $\beta$ is too large, e.g. $\beta>1$ (Fig. 3(c)-(f)). On the other hand, the larger $\mu$ is, the better the sharp tip is captured. However, the captured surface becomes rougher for large $\mu$. Empirically, the best balance happens when $\mu=2000$ and $\beta=1$. Because the parameter $\alpha$ is less important than $\beta$ (as explained at the beginning of Section 2.1) in our experiments, the parameter $\alpha$ is fixed to be 0.01 , the constants $a$ and $b$ in (2.7) are 2 and 4 respectively. The captured brain aneurysm is shown in Fig. 4 with $(\mu=2000, \beta=1)$. Fig. 4 (b) presents the volume of the aneurysm and Fig. 4(c) and 4 (d) show that the area of high mean and Gaussian curvatures locates at the tip of aneurysm. It has been suggested by researchers that certain geometrical characteristics such as a bleb and tip may be associated with aneurysm rupture. Thus the information of mean curvature, 


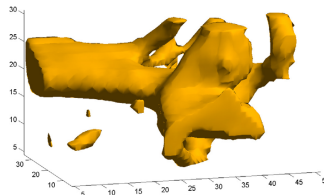

$(a)$

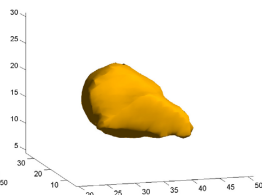

(b)

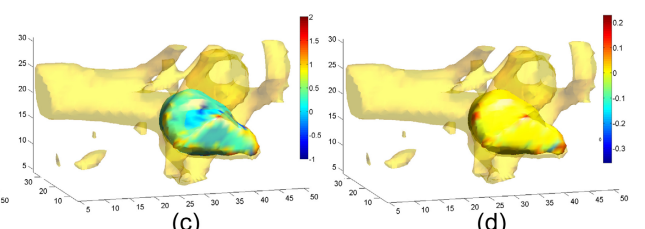

(c)

(d)

Fig. 4. (a) Is the entire structure of the arteries and aneurysm; (b) is the captured aneurysm through our illusory surface algorithm (2.5); (c) and (d) are the mean and Gaussian curvatures of the aneurysm. Both of them show that the curvatures around the tip of the aneurysm are relatively high.

which can indicate the geometry change in value, may provide a new tool to study aneurysm rupture in the future.

\subsection{Validation}

To further test the robustness of our algorithm, we validate the modified surface capturing model by selecting different initial points. Six sets of points indicating the target region (aneurysm) are used to initiate our algorithm. The parameters $\alpha=0.01, \beta=1$, and $\mu=2000$ are used in the algorithm, as determined in the previous section. Results are shown in Fig. 5] The volumes captured by different initial points are $204.5 \pm 8.65 \mathrm{~mm}^{3}$ (mean \pm standard deviation). As a result, we expect the deviation of the volume computation which can be caused by different users is only about $4 \%$ of the total aneurysm volume. Our future work will involve applying this model to different blood vessels and further confirm the accuracy and reproducibility of our algorithm.

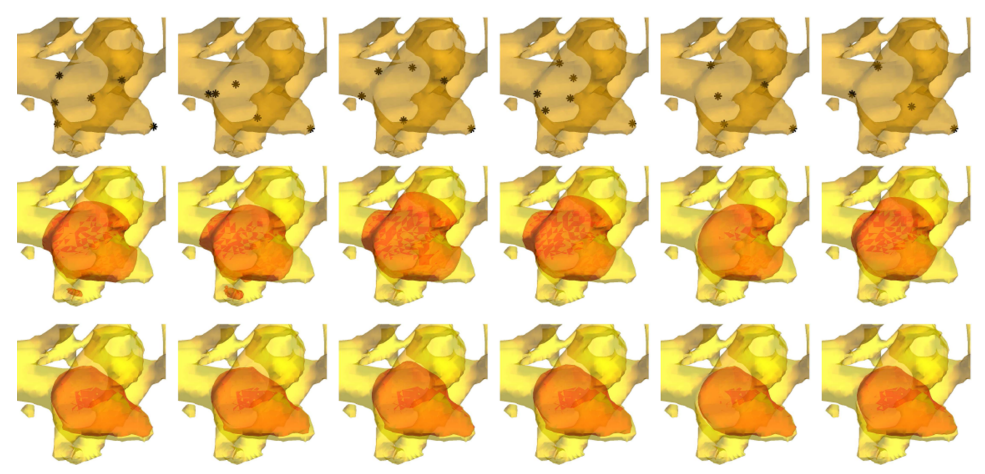

Fig. 5. The resulting volumes for the different points chosen by users from left to right are: $207.375 \mathrm{~mm}^{3}, 205.875 \mathrm{~mm}^{3}, 217.5 \mathrm{~mm}^{3}, 207 \mathrm{~mm}^{3}, 193.875 \mathrm{~mm}^{3}$ and $195.75 \mathrm{~mm}^{3}$. Top row is the given points by users. Middle row is the corresponding initial surfaces. Bottom is the corresponding final captured surfaces. 


\section{Conclusion}

A method to quantify the volume and geometry of brain aneurysms is needed to better study how they associate with aneurysmal growth and rupture. In this paper, we introduced a level set based PDE model to capture and compute the geometry of brain aneurysm. We introduced a supervised strategy with only one parameter $\beta$ and no more than six initial points determined by users to capture surfaces of aneurysms. The numerical results showed that the final surface captured the entire target region and we were able to compute the volume and curvatures for clinical studies. There is huge variation among brain aneurysms and being able to quantify the geometry of irregular shapes is especially important for studying the associations of shape with rupture. Our future work will involve applying this algorithm to diverse aneurysm shapes and improving the algorithm for different clinical purpose.

\section{References}

1. Kassell, N.F., Torner, J.C.: Aneurysmal rebleeding: a preliminary report from the Cooperative Aneurysm Study. Neurosurgery 13(5), 479-481 (1983)

2. Unruptured intracranial aneurysms-risk of rupture and risks of surgical intervention. International Study of Unruptured Intracranial Aneurysms Investigators. N. Engl. J. Med. 339(24), 1725-1733 (1998)

3. Raghavan, M.L., Ma, B., Harbaugh, R.E.: Quantified aneurysm shape and rupture risk. J. Neurosurg. 102(2), 355-362 (2005)

4. Geiger, D., Pao, H.K., Rubin, N.: Salient and Multiple illusory surfaces. In: IEEE Computer Society Conference on Conputer Vision and Pattern Recognition, San Barbara, CA (June 1998)

5. Sarti, A., Malladi, R., Sethian, J.A.: Subjective surfaces: A geometric model for boundary completion. Int. J. Comp. Vision 46(3), 201-221 (2002)

6. Zhu, W., Chan, T.F.: Capture illusory contours: A level set based approach. UCLA CAM Report 03-65 (2003)

7. Yim, P.J., Cebral, J.J., Mullick, R., Marcos, H.B., Choyke, P.L.: Vessel surface reconstruction with a tubular deformable model. IEEE Transactions on Medical Imaging 20(12), 1411-1421 (2001)

8. Chen, J., Amini, A.A.: Quantifying 3-D vascular structures in MRA images using hybrid PDE and geometric deformable models. IEEE Transactions on Medical Imaging 23(10), 1251-1262 (2004)

9. Dong, B., Ye, J., Osher, S.J., Dinov, I.: Level set based nonlocal surface restoration, CAM-Report 07-44 (2007)

10. Tsai, R., Cheng, L.-T., Osher, S.J., Zhao, H.K.: Fast sweeping method for a class of Hamilton-Jacobi equations. SIAM J. Numer. Analy. 41, 673-694 (2003)

11. Osher, S.J., Fedkiw, R.P.: The Level Set Method and Dynamic Implicit Surfaces. Springer, New York (2002)

12. Zhao, H.K., Chan, T.F., Merriman, B., Osher, S.J.: A variational level set approach to multiphase motion. J. Comput. Phys. 127, 179-195 (1996)

13. Peng, D., Merriman, B., Osher, S.J., Zhao, H.K., Kang, M.: A PDE-based fast local level set method. J. Comput. Phys. 155, 410-438 (1999)

14. Goldman, R.: Curvature formulas for implicit curves and surfaces. Computer Aided Geometric Design 22, 632-658 (2005) 